\title{
A rare case of dedifferentiated liposarcoma of the sinonasal cavity: A case report
}

\author{
MASARU MIYAZAKI ${ }^{1,2}$, MIKIKO AOKI ${ }^{1}$, SATORU OBA $^{2}$, \\ TOSHIFUMI SAKATA $^{2}$, TAKASHI NAKAGAWA ${ }^{2}$ and KAZUKI NABESHIMA ${ }^{1}$ \\ Departments of ${ }^{1}$ Pathology and ${ }^{2}$ Otorhinolaryngology and Head and Neck Surgery, \\ Fukuoka University School of Medicine, Fukuoka 814-0180, Japan
}

Received January 31, 2017; Accepted May 19, 2017

DOI: $10.3892 / \mathrm{mco} .2017 .1379$

\begin{abstract}
Sarcoma is an uncommon histopathological presentation of sinonasal tumors, comprising $\sim 15 \%$ of all cases; liposarcoma is particularly uncommon. An analysis of the available medical literature revealed no prior reports of dedifferentiated liposarcoma (DDLPS) of the sinonasal cavity. This case report presents a rare case of DDLPS of the sinonasal cavity. A 40-year old six-week pregnant female was admitted with a left nasal obstruction. Endoscopic evaluation of the left nasal cavity revealed a polypoid lesion. A computed tomography scan indicated a mass invading the left nasal cavity, maxillary sinus and anterior ethmoid sinus with focal destruction of the surrounding bone. A biopsy of the tumor was performed and hematoxylin and eosin staining of the tissue sections revealed proliferation of atypical and pleomorphic spindle cells with enlarged or elongated hyperchromatic nuclei and occasional vacuolated cytoplasm arranged in short interlacing fascicles or storiform structures, accompanied by tumor necrosis. These findings were consistent with undifferentiated pleomorphic sarcoma. Immunohistochemically, the tumor cells were positive for cyclin dependent kinase 4, mouse double minute 2 homolog (MDM2) and adipophilin. Fluorescence in situ hybridization (FISH) analysis revealed amplification of the MDM2 gene. Recently, undifferentiated pleomorphic sarcoma without areas of well-differentiated liposarcoma but with MDM2 amplification is regarded as conventional DDLPS. In the present case, the tumor was diagnosed as a DDLPS due to the results of histopathological, immunohistochemical and FISH analysis.
\end{abstract}

Correspondence to: Dr Mikiko Aoki, Department of Pathology, Fukuoka University School of Medicine, 7-45-1 Nanakuma, Jonan-ku, Fukuoka 814-0180, Japan

E-mail: mikikoss@fukuoka-u.ac.jp

Key words: dedifferentiated liposarcoma, sinonasal cavity, mouse double minute 2 homolog, cyclin dependent kinase 4, adipophilin, fluorescence in situ hybridization

\section{Introduction}

Sinonasal sarcomas comprise only $15 \%$ of all sinonasal malignancies (1). The histopathology of sinonasal sarcomas includes alveolar rhabdomyosarcoma, embryonal rhabdomyosarcoma, unclassified sarcoma, leiomyosarcoma, malignant solitary fibrous tumors, malignant peripheral nerve sheath tumors, synovial sarcoma, angiosarcoma $(2,3)$, pleomorphic liposarcoma, chondrosarcoma, myxofibrosarcoma and epithelioid sarcoma (2). The incidence of liposarcoma of the sinonasal cavity is comparatively less frequent. Only two cases of myxoid liposarcoma and one case of pleomorphic liposarcoma of the sinonasal tract have previously been reported $(2,3)$. According to the World Health Organization classification of tumors of soft tissue tumors and bone (4), the histopathology of liposarcoma is categorized into atypical lipomatous tumor/well-differentiated liposarcoma, dedifferentiated liposarcoma (DDLPS), myxoid liposarcoma, pleomorphic liposarcoma and liposarcoma not otherwise specific (4). The relative incidences of four of these five histopathological subtypes of head and neck liposarcoma are as follows: Myxoid type, $40.0 \%$; well-differentiated type, $26.6 \%$; pleomorphic type, $26.6 \%$; dedifferentiated type, $6.7 \%$ (5). The 5-year survival rate for these subtypes of head and neck liposarcoma is $\sim 87 \%$ for patients who underwent surgery alone, $\sim 75 \%$ for patients who underwent surgery and postoperative radiotherapy, and $0 \%$ for those treated by radiotherapy alone (5). The most common location for a DDLPS is in the retroperitoneum (68.3\%), and the proportion of DDLPSs that occur in the head and neck is $1.3 \%$ (6). Currently, peripheral undifferentiated pleomorphic sarcoma (UPS) without areas of well-differentiated liposarcoma but with amplification of mouse double minute 2 homolog (MDM2) is regarded as conventional DDLPS (7). Similarities in the clinical characteristics, morphology, genomic profile and follow-up of peripheral UPS with MDM2 amplification and of conventional DDLPS suggest that peripheral UPS with MDM2 amplification may be DDLPS (7). DDLPS in the head and neck has been reported to be localized to the cheek (8), larynx (9) and soft tissue (10). A review of the available medical literature revealed no prior reports of a DDLPS in the sinonasal tract. The current case study presents a rare case of DDLPS of the sinonasal cavity, and contends that pathologists must consider the possibility of DDLPS in the nasal cavity when pleomorphic spindle cell tumors are observed. 


\section{Case report}

A 40-year-old female patient attended their clinical practice with a left nasal obstruction that had lasted one month. The patient was subsequently admitted to the Department of Otorhinolaryngology at Fukuoka University Hospital (Fukuoka, Japan) in August 2015 for treatment. Endoscopic evaluation of the left nasal cavity revealed a polypoid lesion, and subsequent radiographic evaluation identified a shadow in the left maxillary sinus. The patient had taken oral medication that had not improved the symptoms. Due to double vision and left visual impairment, the patient was referred to the Department of Otorhinolaryngology at Fukuoka University Hospital in August 2015. The patient had no past medical history and was six weeks pregnant at the time of presentation. On clinical examination, a tumor with a smooth surface was observed in the patient's left nasal cavity (Fig. 1). The patient reported double vision on the left and during upward gaze. Left exophthalmos and skin flare of the left palpebral were documented. A slightly enhanced mass present in the left nasal cavity, maxillary sinus and anterior ethmoid sinus was observed on the contrast-enhanced facial computed tomography scan. Bone destruction was identified in the left medial wall and floor of the orbit, the medial wall of the left maxillary sinus, the nasal septum and the base of the skull. The left orbital contents were shifted to the outside due to the expansion and proliferation of the sinonasal tumors (Fig. 2).

The left sinonasal tumor was biopsied in the outpatient clinic. For histopathological analysis, a 1-cm piece of the tumor was excised using forceps. A hematoxylin and eosin (H\&E) stained tissue section indicated the proliferation of atypical and pleomorphic spindle cells with enlarged or elongated hyperchromatic nuclei, which were arranged in short interlacing fascicles or storiform structures and accompanied by tumor necrosis. The cells exhibited frequent mitotic figures, including abnormal tripolar and multipolar ones. Vacuolation of the cytoplasm was also observed (Fig. 3). Immunohistochemically, the tumor cells were positive for cyclin dependent kinase 4 (CDK4; Fig. 4A), MDM2 (Fig. 4B) and S-100 protein, and were negative for cytokeratin AE1/AE3, cytokeratin CAM5.2, h-caldesmon, desmin, $\alpha$-SMA, myogenin, HMB45 and CD34. The MIB-1 labeling index was $55.2 \%$ (Fig. 4C). In addition, the tumor cells were focally positive for adipophilin (Fig. 4D). The amplification of MDM2 (red) compared with the centromere of chromosome 12 (green) was apparent from the FISH analysis (Fig. 5). Amplification was defined as $>12$ fluorescent signals per cell, as previously described (7). Based on these results, the tumor was diagnosed as a DDLPS. The sinonasal tumor was endoscopically resected as much as possible at Fukuoka University Hospital. The pathological findings of the surgical tissue specimen were similar to those of the biopsy sample; the tumor was primarily composed of atypical spindle cells, though lipoblasts and well-differentiated liposarcoma components were not identified.

\section{Discussion}

To the best of our knowledge, the present case is the first report of DDLPS in the sinonasal cavity. Therefore, it is recommended that DDLPS be considered in differential diagnosis when atypical and pleomorphic spindle cell tumors

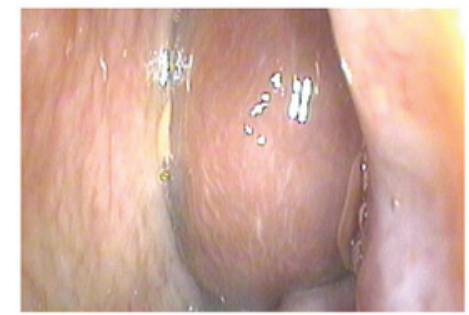

Figure 1. Endoscopic findings. A tumor with a smooth surface in the patient's left nasal cavity is present.
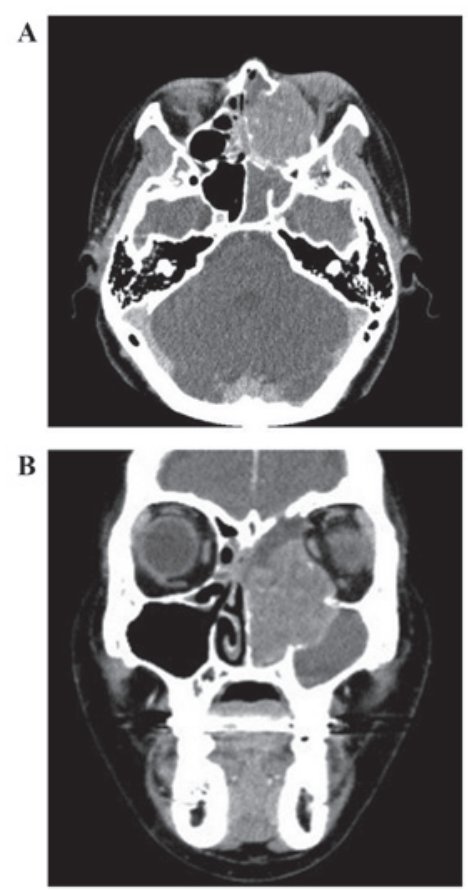

Figure 2. Contrast-enhanced facial CT. (A) The slightly enhanced osteoclastic mass in left anterior ethmoid sinus is visible. Bone destruction in the left medial side and floor of the orbit, medial wall of the left maxillary sinus, nasal septum and skull base were detected. (B) The left orbital contents were shifted to the outside.
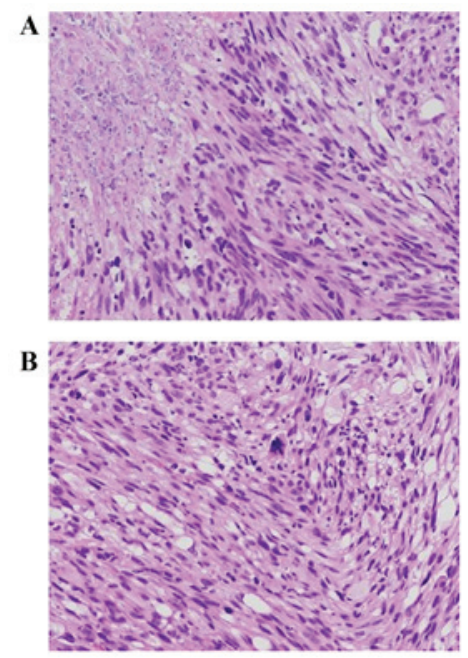

Figure 3. Histopathology using hematoxylin and eosin staining. (A) The section depicts proliferation of atypical and pleomorphic spindle cells with enlarged or elongated hyperchromatic nuclei and vacuolation in the cytoplasm, arranged in short interlacing fascicles or storiform structures and accompanied by necrosis. (B) An abnormal mitotic figure is also visible. 
A

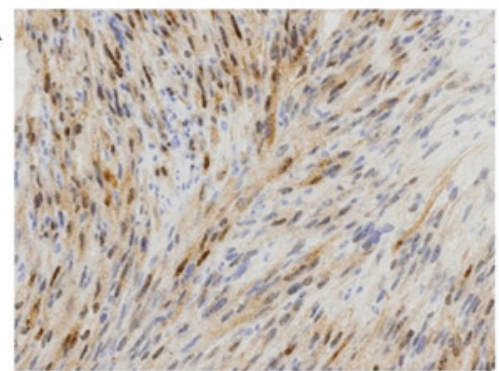

C

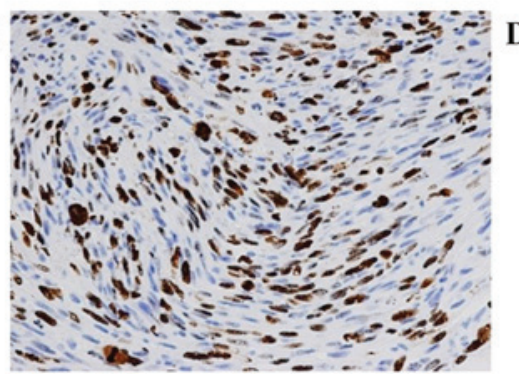

B

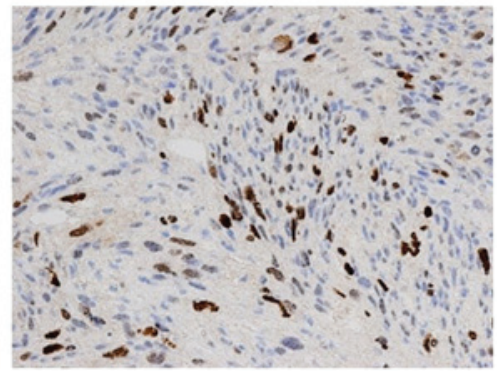

D

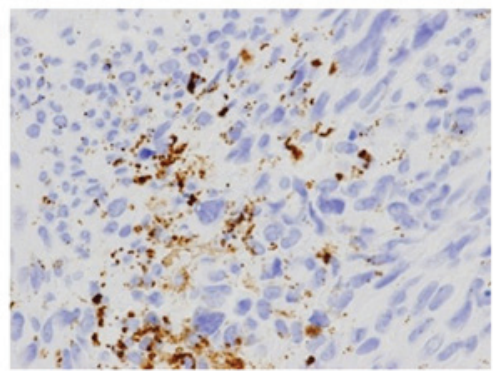

Figure 4. Immunohistochemical analysis. The tumor cells are positive for (A) cyclin dependent kinase 4 and (B) mouse double minute 2 homolog. (C) The MIB-1 labeling index is 55.2\%. (D) Focal positive vesicular staining of the intracellular membrane for adipophilin.

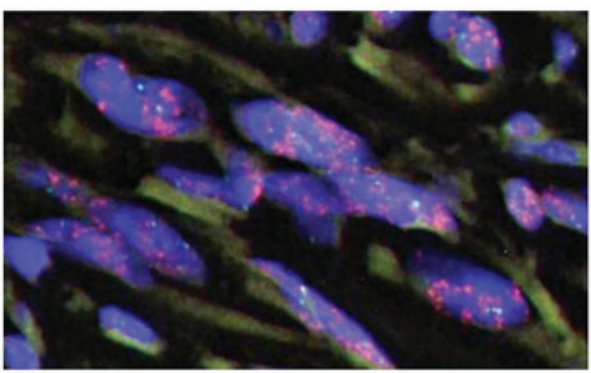

Figure 5. MDM2 FISH analysis. Amplification of MDM2 (red) is indicated, and compared with the paired signals identifying the centromere of chromosome 12 (green). FISH, fluorescence in situ hybridization; MDM2, mouse double minute 2 homolog.

with vacuolated cytoplasm present in the sinonasal cavity are histologically observed. Early diagnosis is crucial for patients with sinonasal malignant tumors in order to improve the prognosis and quality of life (QOL), as the sinonasal cavity is adjacent to vital organs.

In 1979, Evans (11) defined DDLPS as changing from a well-differentiated component to a poorly-differentiated component (i.e., having biphasic findings). The well-differentiated component (atypical lipoblast) was not observed in the tissue biopsy sample in the current case. One possibility is that only the dedifferentiated component was selected during the biopsy. In $\sim 90 \%$ of cases, the dedifferentiated zones have the appearance of high-grade fibrosarcoma or malignant fibrous histiocytoma/UPS (12). Diagnosing a tumor based only on cell morphology is problematic, as the histopathological findings typically include the solid proliferation of pleomorphic and atypical spindle cells. Immunohistochemical staining and analysis is also essential. Overall, $\geq 90 \%$ of DDLPS cases exhibit both MDM2 and CDK4 staining (13). DDLPS frequently has MDM2 amplification, in addition to supernumerary rings and giant marker chromosomes involving 12q13-15 (14). FISH is interpretable in $\sim 90 \%$ of cases; it is more specific and sensitive compared with quantitative polymerase chain reaction and immunohistochemistry (15). Certain sarcoma cases that may occur in the sinonasal tracts harbor MDM2 amplifications in alveolar rhabdomyosarcoma (11\%) (16), anaplastic embryonal rhabdomyosarcoma (14\%) (16), leiomyosarcoma (16), malignant peripheral nerve sheath tumors (20\%) (17), synovial sarcoma (33\%) (18), pleomorphic liposarcoma (33\%) (19) and myxofibrosarcoma (33\%) (19). These types of sarcomas can be excluded using the results of histological and immunohistological analysis. In the current case, the tumor was determined to be positive for adipophilin via immunohistochemistry. Iwasaki et al (20) demonstrated that DDLPS exhibits varying positivity for adipophilin in tumor cells, although the ratio of positive tumor cells to the total number of tumor cells was low. Therefore, the tumor could be diagnosed as DDLPS in the present case, due to the following observations: A pleomorphic and spindle cell tumor with vacuolated cytoplasm determined by H\&E; positivity for MDM2, CDK4 and adipophilin determined using immunohistochemistry; MDM2 amplification revealed by FISH. Therefore, when a tumor is identified in the sinonasal cavity and contains atypical pleomorphic spindle cells with vacuolated cytoplasm, considering every differential diagnosis is necessary.

In patients with sinonasal malignant tumors, early diagnosis is imperative for better prognosis and QOL, as the sinonasal cavity is adjacent to vital organs such as the brain and eyes. The current first-line treatment for a DDLPS is surgery (21). Ensuring a sufficient but safe surgical margin is challenging due to the proximity of the aforementioned organs, and also due to cosmetic aspects. However, the tumor often infiltrates intraorbitally, as revealed in imaging findings, and bone destruction around the skull base is also frequently observed. Ophthalmectomy, maxillary resection and frontal lobe partial resection are often required to ensure a $1-\mathrm{cm}$ safe surgical margin. There is currently no standard treatment strategy for dedifferentiated liposarcoma of the sinonasal cavity, with the aforementioned options being predominantly 
experimental at present. Therefore, major dysfunction and severely decreased QOL due to the effects on the patient's appearance are taken into consideration. An established optimal chemotherapy regimen for liposarcoma has not yet been reported. In the current case, endoscopic sinonasal tumor resection to the maximum extent possible was performed, and subsequent adjuvant radiotherapy was administered as the patient wished to preserve their eyesight and capacity for eye movement. The pathological findings of the surgical tissue specimen were similar to those of the biopsy sample; lipoblasts and well-differentiated liposarcoma section were not identified. Radiotherapy at a total dose of $66 \mathrm{~Gy}$ and six courses of doxorubicin $\left(60 \mathrm{mg} / \mathrm{m}^{2}\right)$ were administered in order to suppress tumor progression. The patient was followed up using endoscopic evaluation each month and a CT scan every two months. No progression of the tumor was observed using these techniques at eight months following postoperative chemotherapy.

\section{References}

1. Hanna E, DeMonte F, Ibrahim S, Roberts D, Levine N and Kupferman M: Endoscopic resection of sinonasal cancers with and without craniotomy: Oncologic results. Arch Otolaryngol Head Neck Surg 135: 1219-1224, 2009.

2. Szablewski V, Neuville A, Terrier P, Laé M, Schaub R, Garrel R, Coindre JM and Costes V: Adult sinonasal soft tissue sarcoma: Analysis of 48 cases from the French Sarcoma Group database. Laryngoscope 125: 615-623, 2015.

3. Thompson SM, Duque CS, Sheth RN, Casiano RR, Morcos JJ and Gomez-Fernandez CR: Case report: Liposarcoma of the sinonasal tract. Br J Radiol 82: e160-e163, 2009.

4. Fletcher CD, Bridge JA, Hogendoorn PCW and Mertens F: WHO Classification of Tumors of Soft Tissue and Bone. 4th edition. IARC, Lyon, 2013.

5. Gritli S, Khamassi K, Lachkhem A, Touati S, Chorfa A, Ben Makhlouf T, El May A and Gammoudi A: Head and neck liposarcomas: A 32 year experience. Auris Nasus Larynx 37: 347-351, 2010.

6. Henricks AH, Chu YC, Goldblum JR and Weiss SW: Dedifferentiated liposarcoma: A clinicopathological analysis of 155 cases with aproposal for an expanded definition of dedifferentiation. Am J Surg Pathol 21: 271-281, 1997.

7. Le Guellec S, Chibon F, Ouali M, Perot G, Decouvelaere AV, Robin YM, Larousserie F, Terrier P, Coindre JM and Neuville A: Are peripheral purely undifferentiated pleomorphic sarcomas with MDM2 amplification dedifferentiated liposarcomas? Am J Surg Pathol 38: 293-304, 2014.

8. de la Roza G, Baredes S and Aisner SC: Dedifferentiated liposarcoma of the cheek. Ann Diagn Pathol 8: 352-357, 2004.

9. Makeieff M, Pelliccia P, Poizat F, Arnaud S, Rat F, Cupissol D, Guerrier B and Costes V: Laryngeal dedifferentiated liposarcoma. Eur Arch Otorhinolaryngol 267: 991-994, 2010.

10. Zreik R, Soyalp K, Ruiz S, Ward R, Dobin S, Chen X, Liu L and Rao A: Ultrasound-guided fine-needle aspiration of a posterior neck dedifferentiated liposarcoma with MDM2 fluorescence in situ hybridization performed on a Pap-stained smear. Diagn Cytopathol 43: 320-324, 2015.

11. Evans HL: Liposarcoma: A study of 55 cases with a reassessment of its classification. Am J Surg Pathol 3: 507-523, 1979.
12. McCormick D, Mentzel T, Beham A and Fletcher CD: Dedifferentiated liposarcoma. Clinicopathologic analysis of 32 cases suggesting a better prognostic subgroup among pleomorphic sarcomas. Am J Surg Pathol 18: 1213-1223, 1994.

13. Binh MB, Sastre-Garau X, Guillou L, de Pinieux G, Terrier P, Lagacé R, Aurias A, Hostein I and Coindre JM: MDM2 and CDK4 immunostainings are useful adjuncts in diagnosing well-differentiated and dedifferentiated liposarcoma subtypes: A comparative analysis of 559 soft tissue neoplasms with genetic data. Am J Surg Pathol 29: 1340-1347, 2005.

14. Sandberg AA: Updates on the cytogenetics and molecular genetics of bone and soft tissue tumors: Liposarcoma. Cancer Genet Cytogenet 155: 1-24, 2004.

15. Sirvent N, Coindre JM, Maire G, Hostein I, Keslair F, Guillou L, Ranchere-Vince D, Terrier P and Pedeutour F: Detection of MDM2-CDK4 amplification by fluorescence in situ hybridization in 200 paraffin-embedded tumor samples: Utility in diagnosing adipocytic lesions and comparison with immunohistochemistry and real-time PCR. Am J Surg Pathol 31: 1476-1489, 2007.

16. Ragazzini P, Gamberi G, Pazzaglia L, Serra M, Magagnoli G, Ponticelli F, Ferrari C, Ghinelli C, Alberghini M, Bertoni F, et al: Amplification of CDK4, MDM2, SAS and GLI genes in leiomyosarcoma, alveolar and embryonal rhabdomyosarcoma. Histol Histopathol 19: 401-411, 2004.

17. Wallander ML, Tripp S and Layfield LJ: MDM2 amplification in malignant peripheral nerve sheath tumors correlates with p53 protein expression. Arch Pathol Lab Med 136: 95-99, 2012.

18. Ito M, Barys L, O'Reilly T, Young S, Gorbatcheva B, Monahan J, Zumstein-Mecker S, Choong PF, Dickinson I, Crowe P, et al: Comprehensive mapping of $\mathrm{p} 53$ pathway alterations reveals an apparent role for both SNP309 and MDM2 amplification in sarcomagenesis. Clin Cancer Res 17: 416-426, 2011.

19. Miura Y, Keira Y, Ogino J, Nakanishi K, Noguchi H, Inoue T and Hasegawa T: Detection of specific genetic abnormalities by fluorescence in situ hybridization in soft tissue tumors. Pathol Int 62: 16-27, 2012.

20. Iwasaki H, Ishiguro M, Nishio J, Aoki M, Yokoyama R, Yokoyama K, Taguchi K and Nabeshima K: Extensive lipoma-like changes of myxoid liposarcoma: Morphologic, immunohistochemical, and molecular cytogenetic analyses. Virchows Arch 466: 453-464, 2015.

21. Kraus DH, Dubner S, Harrison LB, Strong EW, Hajdu SI, Kher U, Begg C and Brennan MF: Prognostic factors for recurrence and survival in head and neck soft tissue sarcomas. Cancer 74: 697-702, 1994 